\title{
Vitiligo and Melasma: A Psychosocio-Cosmetic Challenge in Context of South Asian Countries
}

\section{Anil K Jha ${ }^{1 *}$ and Subekcha Karki ${ }^{2}$}

${ }^{1}$ Founder Chairman Di Skin Hospital and Research Centre Maharajgunj, Kathmandu, Nepal

${ }^{2}$ Assistant Dermatologist to the Chairman, Di Skin Hospital Maharajgunj, Kathmandu, Nepal

*Corresponding author: Jha AK, Founder Chairman Di Skin Hospital and Research Centre Maharajgunj, Professor and Head of Department Nepal Medical College, Attrarkhel, Jorpati, Kathmandu, Nepal, Tel: +9851032694; E-mail: dranilkjha@hotmail.com

Rec date: Jul 28, 2015; Acc date: August 12, 2015; Pub date: August 25, 2015

Copyright: (c) 2015 Jha AK. This is an open-access article distributed under the terms of the Creative Commons Attribution License, which permits unrestricted use, distribution, and reproduction in any medium, provided the original author and source are credited.

\begin{abstract}
Vitligo and melasma are acquired pigmentary disorder of the skin and also the leading cause for dermatologist consultation in South Asian countries like Nepal. Vitiligo vulgaris and melasma are common examples for depigmentary and hyperpigmentary conditions respectively. Even with the presence of multiple proven medical, surgical and combined modalities of managing them the challenges in South Asian countries with Fitzpatrick skin types III-V are the psychosocio-cosmetic morbidities associated with them.
\end{abstract}

\section{Keywords: Vitligo; Melasma; Skin; Corticosteroids}

\section{Introduction}

Vitligo and melasma are acquired pigmentary disorder of the skin and also the leading cause for dermatologist consultation in South Asian countries like Nepal. Clinically vitiliigo is characterised by well circumscribed, depigmented macules and patches secondary to selective destruction of melanocytes in the skin and mucous membrane, whereas melasma is characterized by symmetrical hyperpigmented macules mainly on the face.

\section{Vitiligo}

\section{Epidemiology}

Vitiligo vulgaris is a common clinical-type skin and mucosal disorder observed in Nepal. It can affect all skin types and affects both sexes equally however, female usually acquire the disease earlier than males. It can occur at any age, cases have been reported as early as 6 weeks after birth. Half of the patients develop the disease before the age of 20 years. Onset at an advanced age occurs but its unusual, should raise concern about associated disease; thyroid dysfunction, rheumatoid arthritis, diabetes mellitus, alopecia areata, atopic dermatitis [1-3].

With a global incidence between 1 to $2 \%$, highest incidence has been reported in India and Mexico. It accounted for $2.82 \%$ of the total outpatients seen in two referral hospitals of Nepal. Thus South-Asian countries could be a significant contributor to the global incidence of this disorder [4].

\section{Etiopathogenesis}

Of several pathophysiologic theories the most prominent are autoimmune phenomenon associated with underlying genetic predisposition, neurohormonal and autocytotoxic $[5,6]$.

\section{Presenting features}

Vitiligo presents as well circumscribed, depigmented macules and patches with convex borders, surrounded by normal skin which is slowly progressive, either by centrifugal expansion of current lesion and/ the appearance of new lesion. May remain asymptomatic, may itch and have a propensity to sunburn. Koebner phenomenon develops in areas of friction and/ or trauma. It typically appears in exposed areas; face and hands. Chronic persistent, spontaneous repigmentation is uncommon and occurs in a perifollicular pattern. In some cases begins insidiously in sun-exposed areas during the spring and summer months. Severe sunburn, pregnancy, skin trauma, and emotional stress may precede onset.

According to the area of involvement various types and subtypes of vitiligo are NSV (non segmental vitiligo); focal, mucosal, acrofacial, generalised, universal, SV (segmental vitiligo); focal, mucosal, uni, bi or plurisegmenntal, mixed and unclassified; focal at onset, multifocal, asymmetrical, non-segmental and mucosal [3].

\section{Diagnosis}

Diagnosis can be made clinically, wood lamp, a handled (UV) irradiation device emitting UVA at a wavelength of approximately 365 $\mathrm{nm}$. If required a skin punch biopsy may be done to confirm the diagnosis.

\section{Current Clinical Trends in Vitiligo Treatments}

Although it is relatively resistant to most of the treatments, spontaneous repigmentation occurs in more than $1-25 \%$ of cases. Some studies stated occurrence of repigmentation in $10-20 \%$. Results of recent (since year 2009) clinical studies in vitiligo field are overviewed, with emphasis on their contribution to improved vitiligo management. The review includes all kinds of clinical studies, ranging from double-blind randomised controlled trials with objective assessment of treatment efficiency and evaluation of patient's satisfaction, to pilot trials aiming to preliminary address a question of treatment utility. 
Sun protection with sunscreen helps prevent sunburn and lessen photo damage as well as the chance that Koebner phenomenon that will occur. It also decreases tanning of the uninvolved skin and therefore lessen the contrast with vitiliginous lesions.

Cosmeceuticals such as camouflage products and self-tanning dyes, consist of three steps including application of base cream, and cover with a foundation selected for each patient then dry up with finishing powder. The newer self-tanning creams containing dihydroxyacetone are useful for light-skinned and olive complexioned patients with acral lesions [7].

Topical corticosteroids are useful for localised vitiligo. Class 1 or $2 / 3$ topical steroids showed $<20 \%$ of BSA achieving $75 \%$ repigmentation (6-8 weeks cycle, alternate with tacrolimus). The effectiveness of i.v. methylprednisolone $(8 \mathrm{mg} / \mathrm{kg}$ bodyweight) administered on 3 consecutive days in patients with generalised vitiligo, showed that high-dose glucocorticosteroid pulse therapy may represent a therapeutic option in patients with generalised progressive vitiligo, and should be further evaluated in a prospective, randomised, clinical trial. In a study, $85 \%$ of the patients presenting with progressive disease showed cessation of disease progression after the infusion therapy. Repigmentation was observed in $71 \%$ of the patients with progressive vitiligo [8].

Calcineurin inhibitor like tacrolimus is almost as effective as clobetasol and is better for sensitive areas (eg. eyelids) because, unlike clobetasol, it does not cause skin atrophy. The mean percentages of repigmentation were Clobetasol $49.3 \%$ and Tacrolimus $41.3 \%$. 90\% of patients experienced some repigmentation after 2 months treatment. Tacrolimus was observed to be the most effective on head and neck lesions compared to trunk and extremities, with shorter disease duration and darker skin phototype predicting better response. In addition, response to tacrolimus monotherapy appeared to be better in children. Study on experimentally induced koebnerization stressed a necessity of early treatment during inflammatory phase accompanying koebnerization, both tacrolimus and mometasone furoate were equally efficient in halting lesion spreading, with pimecrolimus being less potent. Tacrolimus in combination with oral prednisolone was efficient in treatment of newly manifested vitiligo. Pimecrolimus was less efficient than topical mometasone in paediatric vitiligo treatment (mean repigmentation $42 \%$ vs. $65 \%$ in interpatient comparision), with pimecrolimus being efficient only on head and neck, whereas mometasone could be used for treatment of any part of the body [9].

It was found that topical tacrolimus at $0.03 \%$ preparation alone or in combination with mid-potency steroid resulted in repigmentation among $60-80 \%$ of cases and also helped stabilize the progression of the lesions.

\section{Vitamin Derivatives}

Studies have reported that the treatment with vitamin D compounds or their combination with ultraviolet light or corticosteroid enhances repigmentation in vitiligo; however, the casual relationship at the cellular and molecular levels has not so far been investigated. Calcipotriol plus photochemotherapy alos appears very promising but certainly requires further evaluation. In a pilot study, topical calcipotriene and betamethasone dipropionate was a promising combination to treat facial vitiligo, but because of lack of control group, the definite conclusion was hard to make [10].

Combination therapies with tacrolimus vs. fluticasone propionate, mometasone furoate and clobetasol propionate in children with general conclusion of similar efficiency of steroid and tacrolimus showed poor results when SV lesion were treated. Combination of topical tacrolimus, targeted NBUVB and systemic prednisolone showed promising results in recently onset UV resistant areas, such as bony prominences of the extremities.

In combination with NBUVB, tacrolimus showed better results compared to placebo. There were potential benefits gained from combination of Monochromatic excimer laser (MEL) and topical tacrolimus to enhance clinical response. Double blind studies show that pimecrolimus $1 \%$ cream combined with NBUVB is superior to placebo, especially for facial lesions. Combining blister roof transplantation (BR) with khellin in liposomes and ultraviolet light (KLUV) in the treatment of recalcitrant vitiligo patches [11-13].

Complementary therapies such as Ginkgo Biloba and leflunomide have immune modulating properties. In a pilot study potency of oral Ginko biloba extract to halt the progression of active vitiligo resulted in a statistically significant cessation of the disease activity. Disease progression was stopped in a 12-week follow up after leflunomide therapy. At our OPD Ginkgo Biloba are also prescribed and have proven to help stabilize the progression of disease to quite an extent. It is a very good alternative to usually prescribed oral steroids.

Pseudocatalase creams with Dead Sea climatotherapy are also compatible with repigmentation. Analysis of a large cohort study of patients revealed that $85 \%$ patients responded to the therapy. Hence, concluded it as an effective treatment modality of vitiligo with relatively few side effects

Topical melagenina 1 and 2 solution is manufactured in Cuba, is an alcoholic extract from human placenta, containing placental lipoprotein fraction. Its active ingredients are melagenina and calcium chloride which works by triggering the production of melanocytes.

Oral L-phenylalanine is a product of Venenzuela, VitilVenz, containing coenzyme Q10, vitamins $\mathrm{C}$ and $\mathrm{E}$ and other natural stimulators of mitochondria was tested in 100 patients and control subjects with positive results. Patients with extensive disease $(>50 \%$ body area) who desire permanent matching of the skin color but for whom repigmentation is not possible can be depigmented with $20 \%$ monobenzyl ether of hydroquinone, 2 times daily for 9-12 months [11-13].

Natural treatments include vitiligo oil compounds such as psoralea Coryli Folia/ breadroot, coconut oil, kala jeera/ nigella/ black cumin, berberis vulgaris/ barberry root/ trailing mahonia. St.John Wort is a commonly used herb for this condition; the herb oil can be applied to the skin. Wild Duckweed (Lemna Minor) works by reactivating melanocytes, bergamot essential oil has substances in it called bergaptenes that have strong photosensitising action may help turn back on melanocytes which are activated by ultraviolet light pepper has direct antioxidant activity against various free radicals and scientists recently discovered that can even stimulate colouration of the skin in an animal model vitiligo.

Phototherapy UV-A, Narrow Band UV-B), Photochemotherapy (psoralen plus UV-A [PUVA]), Psoralen with sunlight (PUVAsol) Phototherapy is an acknowledged first-line option in generalised vitiligo treatment. Topical and systemic PUVA therapy may require 100-300 treatment sessions to achieve complete repigmentation. NBUVB is as efficient as topical PUVA but with fewer adverse effects (given 2-3 X/week with starting dose of $100-250 \mathrm{~mJ} / \mathrm{cm}^{2}$, increased in increments of $10-20 \%$ until mild asymptomatic erythema achieved). 
The major side effects of photochemotherapy are severe phototoxic reaction, blistering, hyperpigmentation, photo-allergic reaction, hyperkeratosis of lesional skin, skin malignancies. NBUVB in can be used in children, pregnant or lactating women and patients with hepatic or kidney dysfunction. The combination of PUVA and calcipotriol is highly effective and works faster and may be used for shortening the therapy with PUVA in the treatment of vitiligo. Oral PUVA using 8-MOP $(0.4-0.6 \mathrm{mg} / \mathrm{kg})$ administered $2 \mathrm{X} /$ week; initial dose of UVA is usually $0.5-1.0 \mathrm{~J} / \mathrm{cm}^{2}$. Visible light ( $635 \mathrm{~nm}$ low energy laser) for SV treatment resulted in 7 out of 14 patients responded to the treatment (response was defined as achieving at least $25 \%$ of repigmentation) thus confirming efficiency of visible light in SV treatment. In our context also NBUVB seems to provide excellent results among patients with generalised, progressive type of vitiligo; however the limitations are there due to frequent hospital visits. In stable, localised vitiligo PUVAsol are showing good results.

\section{Lasers}

The most recent effective and approved therapy for vitiligo is the $308 \mathrm{~nm}$ excimer laser with or without topical calcineurin antagonists. Efficiency was revealed for excimer laser treatment in; children with $50 \%$ of treated lesions achieved $50 \%$ repigmentation, adults $60.4 \%$ of patches repigmented for more than $75 \%$. Besides surgical methods EL might be an option for SV patients. For vitiligo universalis, treatment with topical 4-methoxyphenol and Q-swithced (QS) ruby laser has been effective. It destroys the melanosome in melanocytes and keratinocytes by selective photothermolysis [11-13].

Vitiligo surgeries $[5,14,15]$ such as cosmetic tattooing is used for localised stable vitiligo, especially of the mucosal type. Pigments used in cosmetic tattoo are inert iron oxides that are available in more than 15shades. The colour is implanted into the dermal layer with specialised techniques and cannot be washed off. Of the general tendency, younger patients respond usually better, with facial and trunk lesions repigmenting better than those with acral location. Side effects noted were like occasional perigraft halo, hypertrophic scar, koebnerization, hypo or hyperpigmentation 2 year follow up of 49 patients who underwent aoutologous non-cultured epidermal method to treat paediatric vitiligo.

Dermatosurgical procedures offer a good alternative and option of management in depigmentary disorders, however, it is important to counsel the patients regarding the disease, procedures and their expected outcome, and follow a strict criteria while selecting patients, so that the outcome is satisfactory both for the doctor and the patient. The choice of procedure must be individualised, considering the size of the lesion to be treated, and readily available facilities.

Procedures like punch grafting, split thickness skin grafting, blister suction grafting are inexpensive, relatively simple to perform and thus can be performed as office procedures. These techniques can be modified to minimize know adverse effects and achieve superior results. At our hospital also we have experienced excellent outcome among our patients especially with stable localised vitiligo areas not responding to topical treatments.

Dermatosurgical procedures produce better results when combined with post-operative PUVA and should be advised for all patients. There is scope for improving the technique of split-thickness skin grafting to harvest uniformly thin grafts using simple hand held dermatome. This technique would ensure an inexpensive, time saving procedure with equally good, if not excellent, cosmetic outcome.
Autologous cultured epidermal grafting (CEA) containing only melanocytes or both keratinocytes and melanocytes used either as sheets or cell suspensions have proved to be effective in treating stable vitiligo but are laboratory dependent. This has eliminated the need of TCL and has been adapted to successfully repigment stable vitiligo lesions. With the introduction of autologous cell harvesting devices (Recell Kit) the methodology is further simplified [5-7].

\section{Melasma}

\section{Epidemiology}

Melasma predominantly affects Fitzpatrick skin phototype 3-4 and often lasts for many years after pregnancy. In Southeast Asia, the prevalence has been reported to be as high as $40 \%$ in females and $20 \%$ in males. A study in Western Nepal concluded that sunscreens were the most commonly used medications and the drug therapy was associated with huge economic impact. The exact prevalence of melasma is unknown. Studies from Mexico and Peru found that melasma accounted for 4 to $10 \%$ of new dermatology hospital referrals. It is more common in women than in men and is rare before puberty. It occurs during pregnancy in approximately $25 \%$ of cases with prevalence ranging between 15 and $50 \%$. Although no race is spared, melasma is far more common in women and in dark skinned individuals. It is a common and growing cosmetic concern among Nepalese population with Fitzpatrick skin types III-V.

In a retrospective study conducted in Kathmandu Nepal, melasma composed the third most common dermatological affection. The total population included 3224 new dermatological cases were 235 i.e. $7.29 \%$ cases of melasma were seen over a period of one year; 203 (12.69\%) were females and $32(1.93 \%)$ were males [2].

\section{Clinico-pathological features}

Symmetrical hyperpigmentation appearing as light brown to dark, muddy brown macules and patches on the face, especially the forehead, cheeks, nose, upper lip or chin. There are two basic forms; Epidermal form lesions which enhance when viewed under wood lamp, featuring melanin deposition mainly in the basal and suprabasal layers and dermal form with superficial and deep perivascular melanophages in the dermis which do not enhance implying an increase in dermal melanin content [16].

Dermoscopy can be used for the diagnosis of melasma. Lesions show diffuse reticular pigmentation in various shades of brown with sparing of follicular openings. Dermal melasma shows diffuse dark brown to greyish pseudo reticular pigmentation. Annular, honeycomb and arcuate structures may be seen. A skin biopsy maybe done to confirm the diagnosis if required.

\section{Etiopathogenesis}

Studies have confirmed that both melanocytosis as well as increased melanogenesis are responsible for the hyperpigmentation in melasma. It was found that vascular endothelial growth factor was increased in lesional skin in melasma signifying increased vascularization. This has clinical implications and indicates the need to devise treatment options to target the vascular component in melasma. In a study it was found that combination of TCC and pulsed dye laser (PDL) was more effective than TCC alone [16] which also prevented the relapses [17]. 
Transcriptional analysis in melasma skin samples found that 279 genes were stimulated and 152 were found to be down regulated. Many melanin biosynthesis related genes as well as melanocyte markers such as TYR, MITF, SILV, and TYRP1 were found to be upregulated in melasma skin. Several other genes involved in other biological pathways were found to be affected. These include Wnt pathway modulation genes, genes involved in prostaglandin synthesis and fatty acid metabolism $[18,19]$.

Another interesting finding was the role of $\mathrm{H} 19$ gene in melasma pathogenesis; H19 gene transcribes a noncoding ribonucleic acid (RNA) and is found to be down regulated in melasma lesions. This induces stimulation of melanogenesis and increased transfer of melanin from melanocytes to keratinocytes $[19,20]$.

iNOS and nuclear factor-kappa B pathways have also been found to be implicated in melasma pathogenesis. In a study iNOS expression was found to be increased in melasma lesions [20].

The most affected biological process in melasma is lipid metabolism genes 22, such as peroxisome proliferator-activated receptor alpha (PPAR), arachidonate 15- lipoxygenase, PPAR gamma coactivator 1 alpha, type B (ALXO 15B), diacylglycerol o-acyltransferase 2-like 3 were found to be down regulated due to chronic UV exposure. Another change seen in melasma skin is thinning of the stratum corneum (SC) as found in skin biopsies that show rete ridge flattening and epidermal thinning. SC thinning coupled with disturbed lipid synthesis is responsible for the impaired SC integrity and a delayed barrier recovery rate seen in melasma skin $[21,22]$.

Melanin index, erythema index, and SC hydration were found to be significantly higher in lesional melasma skin as compared to perilesional normal skin. This proves that prominent vascularization accompanies hyperpigmentation [22].

Prolonged UV exposure-induced dermal inflammation and fibroblast activation may upregulate stem cell factors in the melasma dermis, causing increased melanogenesis. Hence these findings emphasize the need for devising treatment options targeting these pathogenetic factors in melasma.

\section{The Melasma area and severity index}

MASI is a reliable measure of melasma severity. Area of involvement and darkness are sufficient for accurate measurement of the severity of melasma and homogeneity can be eliminated [23].

\section{Current Clinical Trends of Melasma Treatments}

Sunscreen plays a vital role for melasma prevention as well as control and affects the overall outcome of any treatment options for patients. Depending upon the skin type and the sun exposure appropriate SPF (sun protection factor) are prescribed and advised to be used on regular basis.

Hydroquinone (HQ) is dihydric phenol that inhibits the conversion of dopa to melanin by inhibiting the tyrosinase enzyme. Other proposed mechanisms of action include the inhibition of RNA and deoxyribonucleic acid synthesis and destruction of melanocytes. HQ is considered the gold standard of treatment for melasma used at a concentration of $2-4 \%$. Its efficacy is enhanced when used in combinations such as Kligman formula ( $5 \% \mathrm{HQ}, 0.1 \%$ tretinoin, and $0.1 \%$ dexamethasone), modified Kligman's formula (4\% HQ, $0.05 \%$ tretinoin and $1 \%$ hydrocortisone acetate), Pathak's (2\% HQ and
0.05-0.1\% tretinoin) and Westerhof's formula (4.7\% N-acetylcysteine, $2 \%$ HQ and $0.1 \%$ triamcinolone acetonide) [24]. Combination of microencapsulated HQ $4 \%$ with retinol $0.15 \%$ and antioxidants has also been tried in melasma with studies showing improvement in disease severity, pigmentation intensity, lesion area and colorimetry assessments. It was found that after 4 weeks once daily treatment, the skin lightening cream (HQ $4 \%$ with 4 brightening actives) was comparable in both efficacy and safety to the well-established TCC ( $4 \% \mathrm{HQ}, 0.05 \%$ tretinoin, $0.01 \%$ flucinolone acetonide) treatment for melisma [24]. One-third of patients experienced local side effects in the form of erythema, peeling of skin and dryness with both the creams [25]. After eight weeks of daily TCC application, subjects were randomized to receive TCC in a twice weekly or tapering regimen (3/ week [1st month], 2/week [2nd month], 1/week [4th month]) for 6 months. They found that twice-weekly regimen showed better effectiveness on postponing relapse in patients with severe melasma. About $53 \%$ of patients were relapse-free after 6 months [26,27].

Tretinoin is widely used for the treatment of melasma as an overthe-counter lightening agent. Considering its efficacy, peeling with tretinoin may improve pigmentation in melasma. It was found that the efficacy of $1 \%$ tretinoin peel was similar to $70 \%$ GA. Post procedure discomfort as expressed by the patients was significantly lower with $1 \%$ tretinoin [28]. The efficacy of $10 \%$ tretinoin peeling mask was found moderate or marked improvement without any adverse events. At 10 weeks, the difference in the average MASI calculated at baseline and after 10 weeks was 2.9 [27]. Several combination products (Hydroquinone + Retinoids + Topical steroids) and commonly used topical agents are Azelaic acid, Kojic acid, Ascorbic acid, Licorice extract.

Tranexamic Acid (TA) is trans-4-(aminomethyl) cyclohexane carboxylic acid, a lysine analog that is in use as an antifibrinolytic agent for over 30 years. It inhibits UV-induced plasmin activity in keratinocytes by preventing the binding of plasminogen to the keratinocytes. This results in less free arachidonic acid and a diminished ability to produce prostaglandins, and this decreases melanocyte tyrosinase activity. TA can interfere with the catalytic reaction of tyrosinase and inhibit melanogenesis [29]. In the treatment of melasma, TA can be used orally, topically or by intradermal microinjection.

As a hemostatic, TA is prescribed in a dose of $1000 \mathrm{mg} 3$ times daily, whereas in the treatment of melasma, it is used in a dose of 250 $\mathrm{mg}$ twice daily [30,31]. TA $250 \mathrm{mg}$ twice daily for 6 months gave excellent result in $10.8 \%$, good in $54 \%$, fair in $31.1 \%$ and poor in $4.1 \%$. Side effects seen were gastrointestinal discomfort and hypomenorrhea. Recurrence was seen in $9.5 \%$ patients [31].

Topically TA incorporated in a liposome gel formulation resulted in $78.2 \%$ of patients with decrease in the melanin index. However, results were not significant as compared with vehicle. Another finding seen in this study was the erythema induced by topical TA [32].

Intradermal TA $4 \mathrm{mg} / \mathrm{ml}$ caused a significant decrease in MASI score (13.22 vs. 9.02 at week 8 and vs. 7.57 at week $12, \mathrm{P}<0.05$ for both) with minimal side effects [33].

The advantage with TA is its good safety profile. Furthermore, it is temperature stable, UV insensitive and does not get oxidized easily; hence it can be used in SLCs. More studies are needed to evaluate its anti-melasma potential. 
Similarly in our current practice the result of patients undergoing treatment with oral tranexamic acid $500 \mathrm{mg}$ once daily dosage is excellent. Improvement is expected and seen with an average of 1 to 3 months period.

Chemical peels using Alpha Hydroxy Acid Peels, salicylic acid peels, Jessner solution (SSA+Lactic Acid+ Resorcinol+Ethanol), 10\%-50\% Trichloroacetic acid (TCA) peels. In current practice and from experience chemical peels do not give satisfactory result, especially because of the skin type of our patients. Chemical peels might have chances of post inflammatory hyper and/or hypopigmentation that are very common in Skin type III-V.

Laser and Light therapies have been used for melasma such as Green light; Flashlamp-pumped PDL (510 nm), frequency doubled Q switched neodymium: Yttrium aluminium garnet-532 nm (QS Nd: YAG), Red light; Q switched ruby (694 nm), Q switched alexandrite $(755 \mathrm{~nm})$ and Near-infrared: QS Nd: YAG (1064 nm). The concept of Fractional lasers has been applied for the treatment of melasma as well. Fractional lasers have been used for the treatment of pigmented lesions, including melasma. QS NdYAG is the most widely used laser for the treatment of melasma. The fluence used is less than $5 \mathrm{~J} / \mathrm{cm}^{2}$, spot size $6 \mathrm{~mm}$, and frequency of $10 \mathrm{~Hz}$. The number of treatment sessions varies from 5 to 10 at 1 -week intervals.

Nowadays, technique called "laser toning" or "laser facial" has become increasingly popular for the treatment of melasma. This involves the use of a large spot size $(6-8 \mathrm{~mm})$, low fluence (1.6-3.5 $\mathrm{J} / \mathrm{cm}^{2}$ ), multiple passed QS $1064 \mathrm{~nm} \mathrm{Nd}$ : YAG laser performed every 1-2 weeks for several weeks [14]. Although good efficacy has been seen with this technique, side effects have also been reported. These include hypopigmentation, depigmentation, rebound hyperpigmentation, physical urticaria, acneiform eruption, petechiae, and herpes simplex reactivation.

Combination of ablative and pigment selective lasers has also been tried in melasma. Ablative lasers remove the epidermis containing excess melanin; this is followed by the use of $\mathrm{Q}$ switched pigment selective laser that can target the dermal melanophages.

Combined ultrapulse $\mathrm{CO}_{2}$ laser and $\mathrm{Q}$ switched alexandrite laser (QSAL) showed significant response compared with QSAL alone [34]. However, side effects in the form of contact dermatitis and hyperpigmentation were observed in few patients, especially in those with dark skin. Hence, the use of combination of lasers should only be used for refractory melisma [34].

On the horizon are treatments using 4-n-Butylresorcinol, a derivative of resorcinol that inhibits tyrosinase and tyrosinase related protein, enzymes in the melanin biosynthetic pathway [35]. Studies have shown good efficacy and safety with 4-n-butylresorcinol in patients with melisma [36]. It was found that liposome-encapsulated 4-n-butylresorcinol $0.1 \%$ cream resulted in a statistically significant reduction $(\mathrm{P}<0.043)$ in the melanin index on the treatment side [37-46].

\section{Conclusion}

Although not a medical challenge in itself, vitiligo and melasma are the most common psychosocio-cosmetic challenge for dermatologists on daily basis. The treatment should not only focus on the symptomatic level but also on the severe social, cosmetic and psychological morbidities associated with it. The challenges are to make the patient as well as the society realize that these are just pigmentary disorders rather than a medical disease in itself. Patients may be reassured that the disorders are not life threatening or do not lead to physical disability, but it is well known that it can lead to drastic psychosocial consequences, especially in our part of the world. It is well understood in regions like Europe and America with skin type 1-2 due to lack of colour contrast vitiligo is not much given concern but melasma and other hyperpigmentary disorders remain common dermatological challenges there also. Counselling is, and remains the backbone of managing these conditions. It has to be well explained how proper sun exposure is essential for vitiligo treatment whereas while managing melasma proper ways of sun protection should be explained well. Proper counselling directly affects the outcome of our management.

\section{References}

1. Jha AK, Pandey SS, Gulati AK, Bansal V, Shukla VK, et al. (1993) Inoculation of a cultured autologous epidermal suspension containing melanocytes in vitiligo. Arch Dermatol 129: 785-786.

2. Jha AK, Benl A, Amit A, Pattern of Skin Diseases In Kathmandu Nepal, Nepal Journal of Dermatology, Venereology and Leprology.

3. Jha AK, Gurung D (2006) Seasonal variation of skin diseases in Nepal: a hospital based annual study of out-patient visits. Nepal Med Coll J 8: 266-268.

4. Jean L (2012) Dermatology. (3rd Edn), Bolognia.

5. JAM ACAD 2011 Dermatology.

6. Jha A K, Pandey SS, Shukla VN (1994) Punch Grafting In Vitiligo. Ind J Dermatology Venerology and Leprology 60: 188-192.

7. Birlea SA, Costin GE, Norris DA (2009) New insights on therapy with vitamin $\mathrm{D}$ analogs targeting the intracellular pathways that control repigmentation in human vitiligo. Med Res Rev 29: 514-546.

8. Ezzedine K, Lim HW, SuzukiT, Katayama I, Hamzavi I, et al. (2012)And on Behalf of the Vitiligo Global Issue Consensus Conference Panellists, Pigment Cell \& Melanoma Research 25: E1-E13.

9. Parsad D, Saini R, Verma N (1998) Combination of PUVAsol and topical calcipotriol in vitiligo. Dermatology 197: 167-170.

10. Wong R, Lin AN (2013) Efficacy of topical calcineurin inhibitors in vitiligo. Int J Dermatol 52: 491-496.

11. National Institute of Health, Department of Health \& Human Services, National Institute of Arthritis and Musculoskeletal and Skin Disease.

12. Korobko IV (2012) Review of current clinical studies of vitiligo treatments. Dermatol Ther 25 Suppl 1: S17-27.

13. Taieb A, Alomar A, Böhm M, Dell'anna ML, De Pase A, et al. (2013) Guidelines for the management of vitiligo: the European Dermatology Forum consensus. Br J Dermatol 168: 5-19.

14. Sarveswari KN (2010) Cosmetic camouflage in vitiligo. Indian J Dermatol 55: 211-214.

15. Mohanty S, Kumar A, Dhawan J, Sreenivas V, Gupta S (2011) Noncultured extracted hair follicle outer root sheath cell suspension for transplantation in vitiligo. Br J Dermatol 164: 1241-1246.

16. Kim EH, Kim YC, Lee ES, Kang HY (2007) The vascular characteristics of melasma. J Dermatol Sci 46: 111-116.

17. Kim NH, Lee CH, Lee AY (2010) H19 RNA downregulation stimulated melanogenesis in melasma. Pigment Cell Melanoma Res 23: 84-92.

18. Passeron T, Fontas E, Kang HY, Bahadoran P, Lacour JP, et al. (2011) Melasma treatment with pulsed-dye laser and triple combination cream: a prospective, randomized, single-blind, split-face study. Arch Dermatol 147: 1106-1108.

19. Kang HY, Suzuki I, Lee DJ, Ha J, Reiniche P, et al. (2011)Transcriptional profiling shows altered expression of wnt pathway- and lipid metabolism-related genes as well as melanogenesis-related genes in melasma. J Invest Dermatol 131: 1692-1700. 
Citation: Jha AK, Karki S (2015) Vitiligo and Melasma: A Psychosocio-Cosmetic Challenge in Context of South Asian Countries. Pigmentary Disorders S3: S3-005. doi:10.4172/2376-0427.S3-005

Page 6 of 6

20. Jo HY, Kim CK, Suh IB, Ryu SW, Ha KS, et al. (2009) Co-localization of inducible nitric oxide synthase and phosphorylated Akt in the lesional skins of patients with melasma. J Dermatol 36: 10-16.

21. Kang WH, Yoon KH, Lee ES, Kim J, Lee KB, et al. (2002) Melasma: histopathological characteristics in 56 Korean patients. Br J Dermatol 146: 228-237.

22. Pandya AG, Hynan LS, Bhore R, Riley FC, Guevara IL, et al. (2011) Reliability assessment and validation of the Melasma Area and Severity Index (MASI) and a new modified MASI scoring method. J Am Acad Dermatol 64: 78-83.

23. Cestari TF, Hexsel D, Viegas ML, Azulay L, Hassun K, et al. (2006) Validation of a melasma quality of life questionnaire for Brazilian Portuguese language: the MelasQoL-BP study and improvement of QoL of melasma patients after triple combination therapy. Br J Dermatol 156 Suppl 1: 13-20.

24. Cook-Bolden FE, Hamilton SF (2008) An open-label study of the efficacy and tolerability of microencapsulated hydroquinone $4 \%$ and retinol $0.15 \%$ with antioxidants for the treatment of hyperpigmentation. Cutis 81: 365-371.

25. Monheit GD, Dreher F (2013) Comparison of a skin-lightening cream targeting melanogenesis on multiple levels to triple combination cream for melasma. J Drugs Dermatol 12: 270-274.

26. Arellano I, Cestari T, Ocampo-Candiani J, Azulay-Abulafia L, Bezerra Trindade Neto P, et al. (2012) Preventing melasma recurrence: Prescribing a maintenance regimen with an effective triple combination cream based on long-standing clinical severity. J Eur Acad Dermatol Venereol. 26: 611-618.

27. Ghersetich I, Troiano M, Brazzini B, Arunachalam M, Lotti T (2010) Melasma: treatment with $10 \%$ tretinoin peeling mask. J Cosmet Dermatol 9: 117-121.

28. Huh SY, Shin JW, Na JI, Huh CH, Youn SW, et al. (2010) Efficacy and safety of liposome-encapsulated 4 -n-butylresorcinol $0.1 \%$ cream for the treatment of melasma: a randomized controlled split-face trial. J Dermatol 37: 311-315.

29. Zhang X, Yang X, Yang H, Yang Y (2003) Study of inhibitory effect of acidum tranexamicum on melanin synthesis. Chin J Dermatovenerol Integr Tradit West Med 2: 227-229.

30. Dunn CJ, Goa KL (1999) Tranexamic acid: a review of its use in surgery and other indications. Drugs 57: 1005-1032.

31. Wu S, Shi H, Wu H, Yan S, Guo J, et al. (2012) Treatment of melasma with oral administration of tranexamic acid. Aesthetic Plast Surg 36: 964-970.

32. Kanechorn Na Ayuthaya P, Niumphradit N, Manosroi A, Nakakes A (2012) Topical 5\% tranexamic acid for the treatment of melasma in
Asians: a double-blind randomized controlled clinical trial. J Cosmet Laser Ther 14: 150-154.

33. Chan NP, Ho SG, Shek SY, Yeung CK, Chan HH (2010) A case series of facial depigmentation associated with low fluence Q-switched 1,064 nm Nd:YAG laser for skin rejuvenation and melasma. Lasers Surg Med 42: 712-719.

34. Angsuwarangsee S, Polnikorn N (2003) Combined ultrapulse CO2 laser and Q-switched alexandrite laser compared with Q-switched alexandrite laser alone for refractory melasma: split-face design. Dermatol Surg 29: 59-64.

35. Kim DS, Kim SY, Park SH, Choi YG, Kwon SB, et al. (2005) Inhibitory effects of 4-n-butylresorcinol on tyrosinase activity and melanin synthesis. Biol Pharm Bull 28: 2216-2219.

36. Khemis A, Kaiafa A, Queille-Roussel C, Duteil L, Ortonne JP (2007) Evaluation of efficacy and safety of rucinol serum in patients with melasma: a randomized controlled trial. Br J Dermatol 156: 997-1004.

37. Jha AK, Gurung D Dermatosurgical Management Of Leukoderma; Punch Grafting Vs. Split-Thickness Skin Grafting.

38. Yaghoobi R, Omidian M, Bagherani N (2011) Vitiligo: a review of the published work. J Dermatol 38: 419-431.

39. Taieb A, Alomar A, Böhm M, Dell'anna ML, De Pase A(2013) Guidelines For The Management Of Vitiligo: the European Dermatology Forum consensus. Br J Dermatol 168: 5-19.

40. de Leeuw J, Assen YJ, van der Beek N, Bjerring P, Martino Neumann HA (2011) Treatment of vitiligo with khellin liposomes, ultraviolet light and blister roof transplantation. J Eur Acad Dermatol Venereol 25: 74-81.

41. Alikhan Ali Vitiligo A Comprehensive Overview.

42. Lee DJ, Lee J, Ha J, Park KC, Ortonne JP, et al. (2012) Defective barrier function in melasma skin. J Eur Acad Dermatol Venereol 26: 1533-1537.

43. Hong SP, Han SS, Choi SJ, Kim MS, Won CH, et al. (2012) Split-face comparative study of $1550 \mathrm{~nm}$ fractional photothermolysis and trichloroacetic acid $15 \%$ chemical peeling for facial melasma in Asian skin. J Cosmet Laser Ther 14: 81-86.

44. Lee JH, Park JG, Lim SH, Kim JY, Ahn KY, et al. (2006) Localized intradermal microinjection of tranexamic acid for treatment of melasma in Asian patients: a preliminary clinical trial. Dermatol Surg 32: 626-631.

45. Sarkar R, Arora P, Garg VK, Sonthalia S, Gokhale N (2014) Melasma update. Indian Dermatol Online J 5: 426-435.

46. Dwari B, Palaian S, Poudel A, Prabhu S (2009) Clinical profile and management pattern of melasma patients in Western Nepal: A Hospital Based Study Internet Journal of Dermatology 7: 1-3. 